\title{
Organization and characterization of a biosynthetic gene cluster for bafilomycin from Streptomyces griseus DSM 2608
}

\author{
Jae Yoon Hwang ${ }^{\dagger}$, Hyo Sun Kim ${ }^{\dagger}$, Soo Hee Kim, Hye Ryeung Oh and Doo Hyun Nam ${ }^{*}$
}

\begin{abstract}
Streptomyces griseus DSM 2608 produces bafilomycin, an antifungal plecomacrolide antibiotic. We cloned and sequenced an 87.4-kb region, including a polyketide synthase (PKS) region, methoxymalonate genes, flavensomycinate genes, and other putative regulatory genes. The 58.5kb of PKS region consisting 12 PKS modules arranged in five different PKS genes, was assumed to be responsible for the biosynthesis of plecomacrolide backbone including 16-membered macrocyclic lactone. All the modules showed high similarities with typical type I PKS genes. However, the starting module of PKS gene was confirmed to be specific for isobutyrate by sequence comparison of an acyltransferase domain. In downstream of PKS region, the genes for methoxymalonate biosynthesis were located, among which a gene for FkbH-like protein was assumed to play an important role in the production of methoxymalonyl-CoA from glyceryl-CoA. Further the genes encoding flavensomycinyl-ACP biosynthesis for the post-PKS tailoring were also found in the upstream of PKS region. By gene disruption experiments of a dehydratase domain of module 12 and an FkbH-like protein, this gene cluster was confirmed to be involved in the biosynthesis of bafilomycin.
\end{abstract}

Keywords: Streptomyces griseus, Bafilomycin, Plecomacrolide antibiotic, Polyketide synthase

\section{Introduction}

Plecomacrolide antibiotic is an unusual class of macrolide antibiotic which has a structural element of 6-membered hemiacetal ring connected to the macrolactone ring by C3-spacer (Dröse and Altendorf 1997). In early 1980s, two macrolides were discovered: one was bafilomycin showing antifungal activity (Werner et al. 1984) and the other concanamycin screened as an immunosuppressive compound (Kinashi et al. 1984). Later some other compounds in this category were further found from nature, including hygrolidin (Seto et al. 1984), setamycin (Otoguro et al. 1988), leucancidin (O'Shea et al. 1997), micromonospolide (Ohta et al. 2001), and so on.

In early days, plecomacrolide antibiotics were found to have a wide spectrum of activities including antifungal, antimalarial and antiparasitic activity as well as antibacterial activity against Gram-positive bacteria. Since

\footnotetext{
*Correspondence: dhnam@ynu.ac.kr

${ }^{\dagger}$ Equal contributors

College of Pharmacy, Yeungnam University, Dae-dong, Gyongsan 712-749, Korea
}

Bowman et al. (1988) reported that bafilomycin shows a high-affinity inhibitory function on vacuolar-type ATPase (V-ATPase), this compound became a widely-used biochemical research tool to study the function of this type of ATPases. Interestingly, this class of antibiotics makes a clear distinctive inhibition of V-ATPase activity from other type of ATPases (P-ATPase and F-ATPase), which can promote the accumulation of autophagic vacuoles and trigger Bax-dependent autophagy (Shacka et al. 2006). This specific inhibitory function suggests the possibility of clinical application in the treatment of osteoporosis associated with excessive bone resorption (Farina and Gagliardi 2002; Xu et al. 2007).

The unusual structure of plecomacrolide antibiotics evokes researcher's interests about their biosynthetic pathway. Schumann and his collegues $(2004 ; 2007)$ reported the biosynthetic origin of bafilomycin and concanamycin by feeding experiments with ${ }^{13} \mathrm{C}$-labelled precursors. They assumed that the main backbone of plecomacrolide including macrolactone and hemiacetal ring is assembled from an isobutyrate starter unit, 7 propionate extender 
units, 2 acetate extender units, and 2 methoxyl $\mathrm{C}_{2}$ extender units, on the modular type I polyketide synthases (PKSs). They proposed that the methoxymalonyl CoA for methoxyl $\mathrm{C}_{2}$ extender units could be derived from glycerol through D-1,3-bisphosphoglycerate.

The first plecomacrolide antibiotic of which the biosynthetic gene cluster was reported is concanamycin A of Streptomyces neyagawaensis (Haydock et al. 2005). In this cluster, genes for a set of polyketide synthases encoding 14 modules and for deoxysugar biosynthesis were found. Recently, the genome sequence of Kitasatospora setae producing bafilomycin has been revealed (Ichikawa et al. 2010). Among 24 gene clusters for secondary metabolite biosynthesis, the predictive gene cluster for bafilomycin biosynthesis was located in the left subtelomeric region. However, the authors did not further characterize this gene cluster beyond indentifying its putative gene sequences.

Here we report the full gene cluster for bafilomycin biosynthesis from Streptomyces griseus DSM 2608, which was formerly reported as Tü 1922 (Werner et al. 1984; Hagenmaier et al. 1985). The involvement of this gene cluster in bafilomycin biosynthesis was also confirmed by gene disruption experiments.

\section{Materials and methods}

\section{Strains, vectors, culture media and reagents}

Escherichia coli XL1-blue, E. coli XL1-blue MRF' and E. coli JM109 grown on Luria-Bertani (LB) medium at $37^{\circ} \mathrm{C}$ were used routinely for gene manipulation in this study. The cloning vectors pGEM-T-easy, pUC18 or pUC19 plasmid were generally employed for subcloning. For blue/white selection, $0.1 \mathrm{mM}$ of isopropyl-D-thiogalactopyranoside (IPTG), and $80 \mu \mathrm{g} \mathrm{ml}^{-1}$ of 5-bromo-4-chloro-3-indolyl- $\beta$ $\mathrm{D}$-galactopyranoside (X-Gal) were added on the medium, and for positive selection, appropriated antibiotic solutions were also supplemented. In gene disruption experiment, E. coli BW25113/pIJ790 cultured on SOC medium ( $2 \%$ trypton, $0.5 \%$ yeast extract, $0.05 \% \mathrm{NaCl}, 2.5 \mathrm{mM} \mathrm{KCl}$, $10 \mathrm{mM} \mathrm{MgCl} 2,20 \mathrm{mM}$ glucose, $\mathrm{pH}$ 7.0) was used for homologous double-crossover recombination (Datsenko and Wanner 2000) and E. coli ET12567/pUZ8002 grown on LB medium for conjugation (Biermann et al. 1992). The induction of an ara promoter in pIJ790 plasmid was achieved by the addition of $10 \mathrm{mM}$ arabinose to LB agar medium, and pIJ773 plasmid was used as a template for the construction of disruption cassette. The bafilomycinproducer S. griseus DSM 2608 was cultivated on tryptic soy broth (TSB) at $28^{\circ} \mathrm{C}$ for 3 days for simple culture, or mannitol soy flour (MS) agar (2\% mannitol, $2 \%$ soy flour, $2 \%$ agar) for the selection of gene disruptants. The fermentation of this strain was carried out on the production agar medium ( $2 \%$ glucose, $1 \%$ soluble starch, $0.1 \%$ meat extract, $0.4 \%$ yeast extract, $2.5 \%$ soybean meal, $0.2 \% \mathrm{NaCl}$,
0.005\% $\mathrm{K}_{2} \mathrm{HPO}_{4}$, pH 7.3) (Moon et al. 2003). The antifungal activity was measured using Rhizoctonia solani AG-1 (KACC 40401) grown on potato dextrose agar medium. The authentic bafilomycin $A_{1}$ was purchased from Fermentek (Israel) and bafilomymycin $\mathrm{B}_{1}$ from Santa Cruz Biotech (USA).

\section{Preparation of probes for gene screening}

For screening the bafilomycin biosynthetic gene cluster, three different gene fragments of ketosynthase (KS), aminolevulinate synthase (ALS) and FkbH-like protein were amplified from S. griseus chromosomal DNA based on their conserved domains. The chromosomal DNA of $S$. griseus DSM 2608 was isolated from mycelia following the procedure using $10 \%$ cetyltrimethylammonium bromide (CTAB) (Kieser et al. 2000). The primers used for gene amplification by polymerase chain reaction (PCR) were as follows : KS-F (5'-SBVBTTCGACGCSBSSTTCTTCG-3'), KS-R (5'-RCSAGSGASGASGAGCASGCSGTGTC-3'), A LS-F (5'-AGGAACTAGCGGATCTGCAC-3'), ALS-R1 (5'-ACGAAGATCGA GACGATGTG-3'), FkbH-F (5'-A ARTGYCTSGTCTGGGACCTSGACRACACMCTSTG GC-3'), and FkbH-R (5'-GCGTTCATCTGGCTGGTGC GCAGGGTSAGTTCCTCGACCC-3'). The extension of the biosynthetic gene cluster, a new primer set was also designed as follows: ACL-F (5'-GAGGGGGAAGGCG TCCACGAACTCCACCCGGCGCGGGTACTTGTA-3') and ALS-R2 (5'-AATTTTCCGGCGAGCCGGCCCAGT TCGAGGAACTCCCGTTTCGCAT-3'). A typical amplification conditions were as follows: an initial hot start at $94^{\circ} \mathrm{C}$ for $5 \mathrm{~min} ; 35$ cycles of amplification with each repeat of denaturation at $30 \mathrm{sec}$ at $94^{\circ} \mathrm{C}$, annealing at $52.5^{\circ} \mathrm{C}$ for $30 \mathrm{sec}$, and elongation at $72^{\circ} \mathrm{C}$ for $30 \mathrm{sec}$; and final completion at $72^{\circ} \mathrm{C}$ for $5 \mathrm{~min}$. The amplified probes were radiolabeled with $\alpha-\left[{ }^{32} \mathrm{P}\right]$-dCTP $\left(10 \mathrm{mCi} \mathrm{ml}^{-1}\right.$; Perkin Elmer, USA) by DecaLabel ${ }^{\mathrm{TN}}$ DNA labeling kit (Thermo Scientific, UK), and purified by passing through a Elutip-D column (Schleicher \& Shuell, Germany).

\section{Construction and screening of cosmid library of genomic DNA}

The obtained chromosomal DNA was partially digested with $M b o \mathrm{I}$, and the DNA fragments larger than $23 \mathrm{~kb}$ were excised from agarose gel and then ligated with BamHI-digested and dephosphorylated SuperCos1 cosmid vector. The ligated DNA products were then in vitro packaged using Gigapack III packaging extract (Stratagene, USA). After adding SM buffer (phage dilution buffer), the ligated cosmid DNAs (ca $5 \mu \mathrm{g}$ ) were then transfected into E. coli XL1-blue MRF' grown overnight on LB broth supplemented with $10 \mathrm{mM} \mathrm{MgSO}$. The host cells were then incubated on LB liquid media containing ampicillin. The appeared colonies were transferred to Nylon membrane by placing it on the agar plate, and subjected to in 
situ hybridization with ${ }^{32}$ P-labeled probes (Sambrook et al. 1989).

\section{DNA sequencing and analysis}

The selected recombinant cosmids were sent to Genotech Co., Inc. (Daejeon, Korea) for sequencing. The location of the open reading frames (ORFs) in the sequences was determined using FramePlot version 2.3.2 (http://www0.nih.go.jp/ jun/cgi-bin/frameplot.pl) (Ishika wa and Hotta 1999). The homology search with the obtained DNA or protein sequences was performed with BLAST program (http://blast.ncbi.nlm.nih.gov). The multiple alignments of DNA or protein sequences were performed with EBI-ClustalW2 program (http://www. ebi.ac.uk/Tools/clustalw2/index.html).

\section{Gene disruption}

The gene disruption was performed following the protocol of John Innes Centre (Gust et al. 2003). Employing pIJ773 plasmid as a template, the disruption cassette for a dehydratase domain of module 12 was amplified using forward primer ( $5^{\prime}$-GCCTGCGGGTCGACTGGGAGCG GCTGTTCGCGGGGACCATTCCGGGGATCCGTCGA CC-3') and reverse primer (5'-GGTGTTCGAGCTGGG CCGCGGCGAGCATGCCCCGAACGGTGTAGGCTG GAGCTGCTTC-3'), and that for a FkbH-like protein was also amplified using forward primer (5'-AGCGGGC CCGCGTCCGACGAGACGAGGAAGGACGACATGA TTCCGGGGATCCGTCGACC-3') and reverse primer (5'-CGGGCCGGAGCCCGGGAGCACCGGCCGCCGC GGCGGTCATGTAGGCTGGAGCTGCTTC-3'). These extended apramycin disruption cassettes were introduced by electroporation into cell suspension of $E$. coli BW25113/pIJ790 harboring pSGB20 cosmid, which were grown in the presence of arabinose. After incubation overnight at $37^{\circ} \mathrm{C}$, a single clone was selected in which the target gene was replaced by the disruption cassette. For conjugal transfer to bafilomycin-producing S. griseus, the PCR-targeted disruption cosmid was purified and transformed in E. coli ET12567/pUZ8002 by electroporation, and then transferred by intergeneric conjugation to wild strain. The conjugation was tried using Streptomyces spores obtained from MS agar plate and then heated at $50^{\circ} \mathrm{C}$ for $10 \mathrm{~min}$ to induce germination. The mixture of E. coli ET12567/pUZ8002 cells and heated spores were spread on MS agar plate supplemented with $10 \mathrm{mM} \mathrm{MgCl}$ and apramycin at $30^{\circ} \mathrm{C}$ overnight. One day after, the plates were overlaid with 300 $\mu \mathrm{L}$ of apramycin and nalidixic acid and incubated for 5 days more. For selection of the disrupted colonies, replicaplates with single colonies were made onto a nutrient agar containing nalidixic acid and apramycin for positive selection, and the other onto a nutrient agar containing nalidixic acid, apramycin and kanamycin for negative selection.

\section{Metabolite analysis}

The wild strain and the gene disuptants were cultured on the production agar plates $(\mathrm{pH} 7.3)$ at $28^{\circ} \mathrm{C}$ for 7 days. The plates were minced and directly extracted twice with ethyl acetate. After drying on anhydrous sodium sulfate and concentration in vacuo, the extracts dissolved in

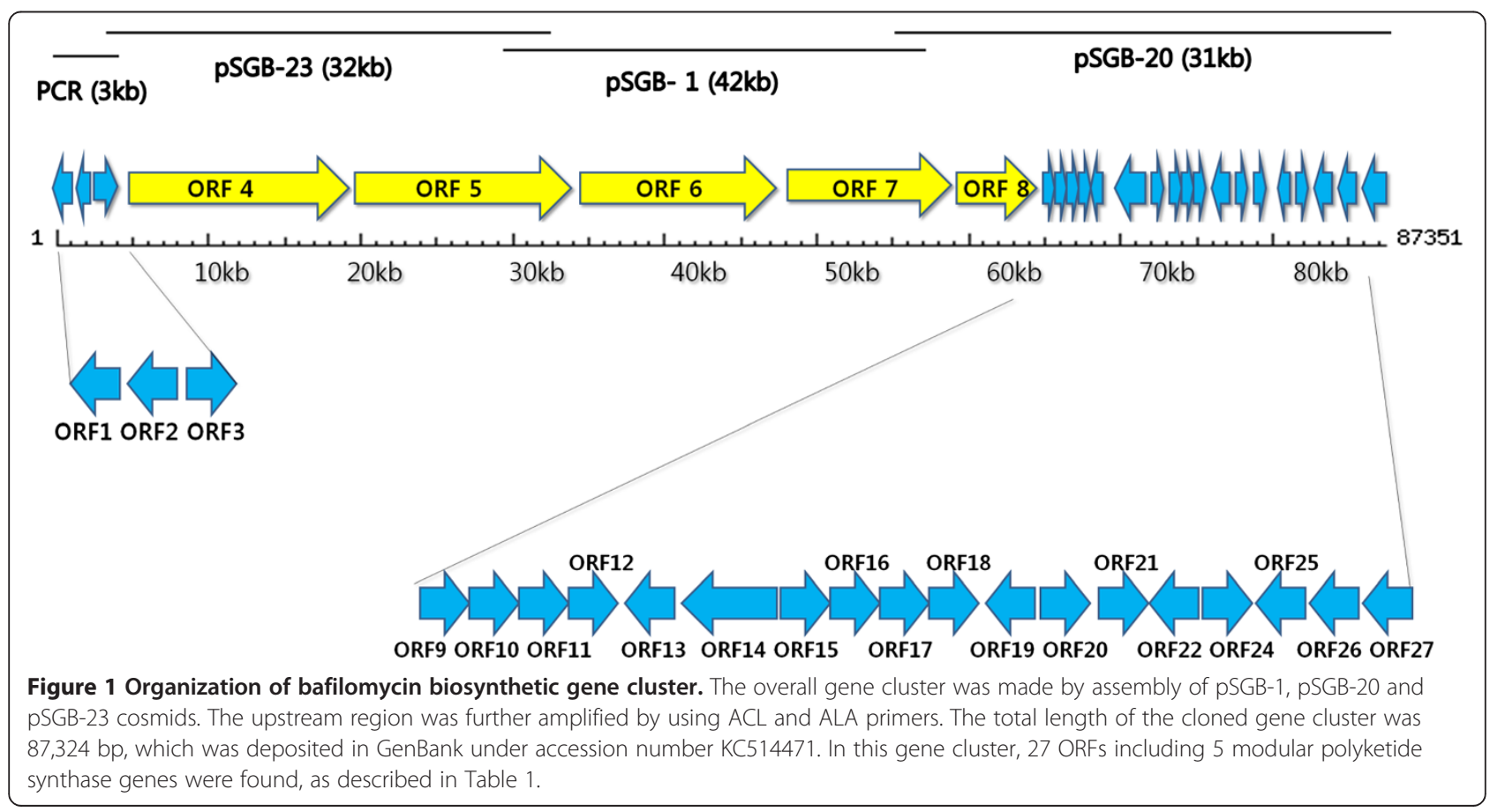


Table 1 Gene organization of bafilomycin biosynthetic gene cluster of Streptomyces griseus DSM 2608

\begin{tabular}{|c|c|c|c|c|c|c|}
\hline ORF & $\begin{array}{l}\text { Gene } \\
\text { product }\end{array}$ & $\begin{array}{l}\text { Number } \\
\text { of amino } \\
\text { acid }\end{array}$ & Deduced function & $\begin{array}{l}\text { The closest gene } \\
\text { (GenBank number) }\end{array}$ & Gene strain & $\begin{array}{l}\text { Homology } \\
(\%)\end{array}$ \\
\hline ORF1 & BafBI & 460 & acyl-CoA ligase (partial) & ADC79613.1 & Streptomyces lohii & 93 \\
\hline ORF2 & BafBII & 513 & amide synthetase & ADC79614.1 & Streptomyces lohii & 96 \\
\hline ORF3 & BafBIII & 415 & 5-aminolevulinate synthase & ADC79615.1 & Streptomyces lohii & 98 \\
\hline ORF4 & BafSI & 5016 & modular polyketide synthase I & ADC79616.1 & Streptomyces lohii & 93 \\
\hline ORF5 & BafSII & 5089 & modular polyketide synthase II & YP_004909056.1 & Kitasatospora setae & 85 \\
\hline ORF6 & BafSIII & 3981 & modular polyketide synthase III & ADC79618.1 & Streptomyces lohii & 94 \\
\hline ORF7 & BafSIV & 3627 & modular polyketide synthase IV & ADC79619.1 & Streptomyces lohii & 89 \\
\hline ORF8 & BafSV & 2106 & modular polyketide synthase $\mathrm{V}$ & ADC79620.1 & Streptomyces lohii & 93 \\
\hline ORF9 & BafAl & 297 & $\begin{array}{l}\text { 3-hydroxyacyl-CoA dehydrogenase } \\
\text { (NAD-dependent) }\end{array}$ & ADC79621.1 & Streptomyces lohii & 97 \\
\hline ORF10 & BafAll & 94 & acyl carrier protein & ADC79622.1 & Streptomyces lohii & 97 \\
\hline ORF11 & BafAlll & 364 & acyl-CoA dehydrogenases (FAD-dependent) & ADC79623.1 & Streptomyces lohii & 98 \\
\hline ORF12 & BafAlV & 367 & FkbH-like protein & ADC79624.1 & Streptomyces lohii & 97 \\
\hline ORF13 & BafAV & 221 & O-methyltransferase & ADC79625.1 & Streptomyces lohii & 95 \\
\hline ORF14 & BafRl & 610 & AfsR family transcriptional regulator & ADC79626.1 & Streptomyces lohii & 96 \\
\hline ORF15 & Baft & 254 & Thioesterase II & ADC79627.1 & Streptomyces lohii & 95 \\
\hline ORF16 & BafRII & 118 & LuxR-like protein & ADC79628.1 & Streptomyces lohii & 91 \\
\hline ORF17 & BafCll & 321 & malonyl transferase & ADC79629.1 & Streptomyces lohii & 97 \\
\hline ORF18 & $\mathrm{BafCl}$ & 360 & Putative CoA ligase & ADC79630.1 & Streptomyces lohii & 97 \\
\hline ORF19 & & 511 & XRE family transcriptional regulator & ZP_04709597.1 & Streptomyces roseosporus & 98 \\
\hline ORF20 & & 506 & 2-methylcitrate reductase & ZP_04709598.1 & Streptomyces roseosporus & 95 \\
\hline ORF21 & & 379 & methylcitrate synthase & ZP_04709599.1 & Streptomyces roseosporus & 94 \\
\hline ORF22 & & 224 & GntR family transcriptional regulator & ZP_11438254.1 & Mycobacterium abscessus & 53 \\
\hline ORF23 & & 285 & methylcitrate lyase & ZP_04709600.1 & Streptomyces roseosporus & 91 \\
\hline ORF24 & & 491 & putative transcriptional regulator & ZP_19189491.1 & Streptomyces ipomoeae & 71 \\
\hline ORF25 & & 846 & Osmosensitive K+ channel histidine kinase & ZP_04995839.1 & Streptomyces sp. Mg1 & 84 \\
\hline ORF26 & & 225 & $\mathrm{~K}^{+}$-transporting ATPase subunit $\mathrm{C}$ & ZP_04710866.1 & Streptomyces roseosporus & 82 \\
\hline ORF27 & & 486 & $\mathrm{~K}^{+}$-transporting ATPase subunit B (partial) & YP_001824190.1 & Streptomyces griseus subsp. griseus & 91 \\
\hline
\end{tabular}

chloroform-methanol (9:1) were chromatographed on Kieselgel 60 (0.063 0.200 mm, Merck, Germany) with the same solvent. The fractions of wild-type showing the antimicrobial activity against $R$. solani were collected. Once more purification was performed by chromatography on Lichroprep Si 60 (40-63 $\mu \mathrm{m}$; Merck, Germany) with the solvent system of chloroform-methanol (95:5), and the antimicrobial fractions were also collected. In case of disruptants, the fractions corresponding to the same elution number were collected. The fractions were dried and applied on high-performance liquid chromatography (SCL-10A $A_{V P}$ system, Shimadzu Co., Japan) using YMCPack Pro $\mathrm{C}_{18}$ reverse-phase column $(250 \mathrm{~mm} \times 4.6 \mathrm{~mm}$. D., S-5 $\mu \mathrm{m}, 12 \mathrm{~nm}$ ) (YMC Co., Ltd., Japan). The isocratic mobile phase of acetonitrile-methanol (9:1) was pumped at a flow rate of $1 \mathrm{ml} \mathrm{min}^{-1}$, and the eluent was detected at $254 \mathrm{~nm}$.

\section{Results}

Screening of a cosmid library of S. griseus genomic DNA

As screening probes, the DNA fragments of KS, ALS and FkbH-like protein were firstly amplified from S. griseus DSM 2608 chromosomal DNA. The gene amplification yielded DNA fragments having about 590 bp for KS gene, $520 \mathrm{bp}$ for ALS gene and $600 \mathrm{bp}$ for FkbH-like protein gene, respectively (Additional file 1: Figure S1). The amplified KS probe had a highly similar nucleotide sequence over 95\% with TrdAIII gene of Streptomyces sp. SCSIO1666 (GenBank No. HQ852227.1) and TamAIII gene of Streptomyces sp. 307-9 (GenBank No. GU 


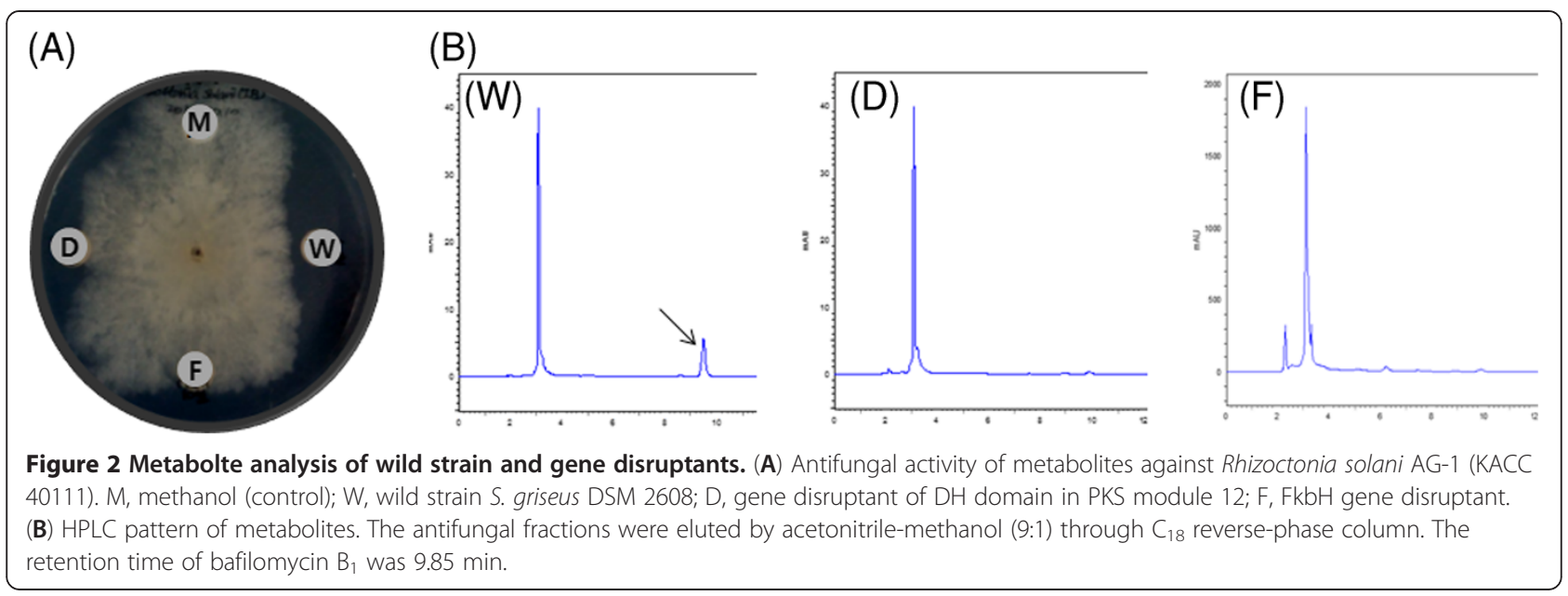

385216.1). The amplified ALS probe also showed the high homology around $92 \%$ in nucleotide sequences with $b \mathrm{fmK}$ gene of Kitasatospora setae KM-6054 (GenBank No. NC_016109.1) and bafZ gene of S. lohii (GenBank No. GU390405.1). The amplified probe for FkbH-like protein fragment also exhibited the high homology over $90 \%$ in nucleotide sequences with $b f m E$ gene of $K$. setae KM6054 (GenBank No. NC_016109.1) and bafE gene of $S$. lohii (GenBank No. GU390405.1).

The cosmid library was made in SuperCos1 vector at BamHI site by ligating the large fragments of chromosomal DNA partially digested with MboI. The recombinant cosmid were in vitro packaged and transfected into $E$. coli XL1-blue MRF' strain. Around $5 \times 10^{4}$ to $5 \times 10^{5}$ transfected bacterial colonies per $\mu \mathrm{g}$ DNA were counted on LB plate containing ampicillin. The obtained cosmid clones were diluted $10^{4}-10^{6}$ fold to give around $5 \times 10^{3}$ colonies per LB plate, and screened the bafilomycin biosynthetic gene cluster using the amplified probes labeled with $\alpha-\left[{ }^{32} \mathrm{P}\right]-\mathrm{dCTP}$.

One positive clone, pSGB1 was firstly screened based on the multiple strong signals with KS probes, which indicates the presence of type I multi-modular PKS responsible for the biosynthesis of type I polyketide molecule (Additional file 1: Figure S2). To extend the biosynthetic

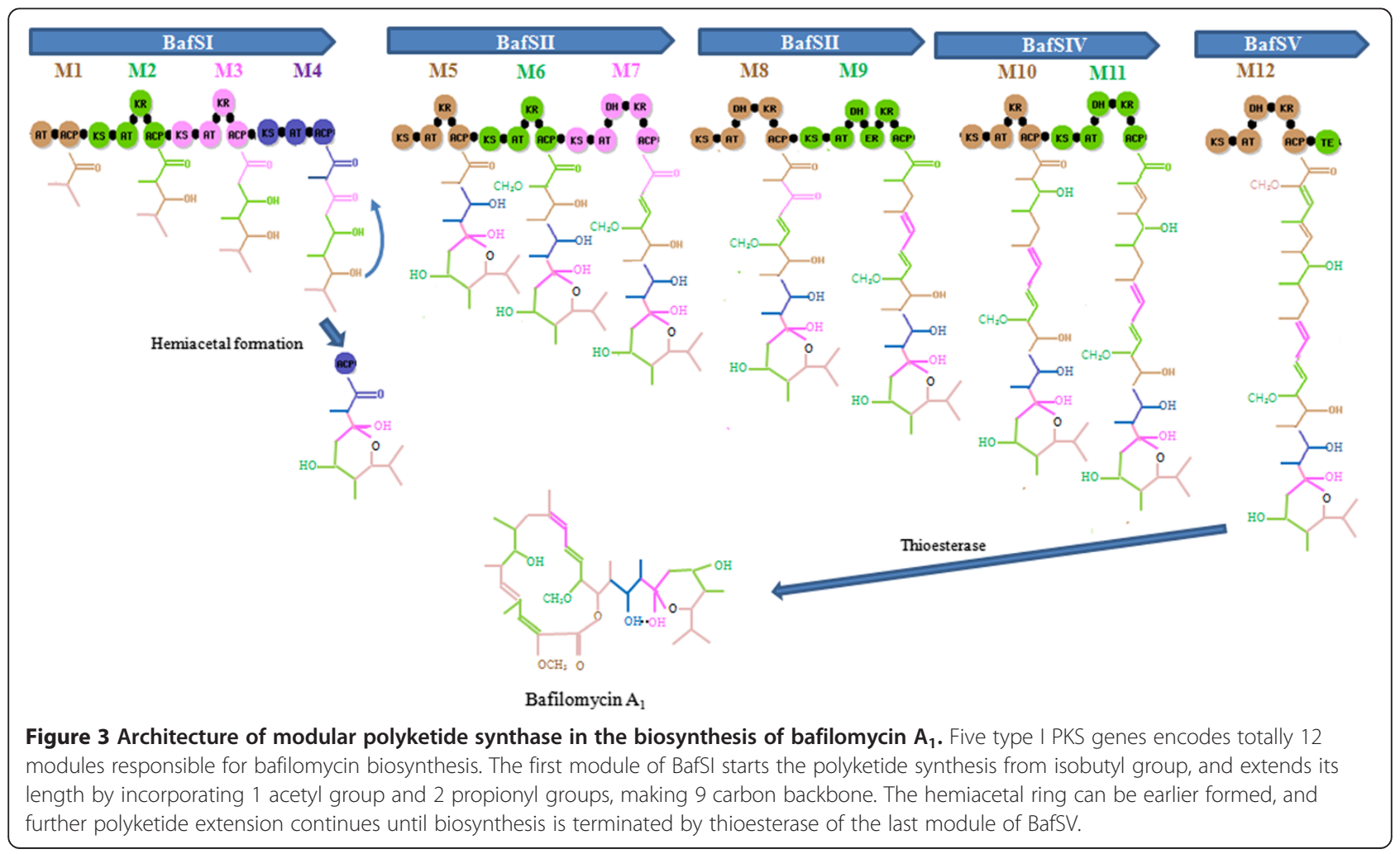


gene cluster in pSGB1 cosmid by chromosome walking, the amplified DNA fragments of ALS gene and FkbHlike protein gene were employed as the second probes for colony hybridization. Among 63 positive cosmid clones screened using KS probes, only 2 cosmid clones were hybridized with ALS probe and only 3 clones with FkbH probe during the second screening. As shown in Additional file 1: Figure S2, the chosen cosmid clones, pSGB23 and pSGB20, gave a strong signal with ALS probe or $\mathrm{FkbH}$ probe in addition to KS probe.

\section{Gene organization of bafilomycin biosynthetic gene cluster}

By overlapping and combining the nucleotide sequences of pSGB-1 (42 kb), pSGB-20 (34 kb), and pSGB-23 cosmid clones (31 kb), a contiguous $87.4 \mathrm{~kb}$ genetic locus encoding 27 ORFs was drawn up as a complete gene cluster for bafilomycin biosynthesis (Figure 1). The putative functions of 27 ORFs present in this contiguous sequence were analyzed by BLAST search (Table 1). The gene sequence was submitted as GenBank accession number KC514471.

Five genes for BafSI to BafSV were deduced to be the type I PKS genes responsible for the biosynthesis of main macrolactone backbone of bafilomycin. At the upstream of gene cluster, three genes for BafBI to BafBIII were presumed to be engaged in the biosynthesis of flavensomycinyl moiety of bafilomycin $\mathrm{B}_{1}$. At the downstream of PKS genes, the genes involved in the biosynthesis of methoxymalonyl-CoA from glycerol as a polyketide precursor (Chan and Thomas 2010) were also found from BafAI to BafAV. Two genes for transcription regulators in the families of SARP and LuxR were also found at the downstream of gene cluster. However, it is uncertain whether ten gene products located at downstream of gene cluster are related to bafilomycin biosynthesis or not.

\section{Functional characterization of bafilomycin biosynthetic gene cluster}

In order to confirm whether the cloned gene cluster is really involved in the biosynthesis of bafilomycin, the gene for a dehydratase domain of module 12 (DH12) of PKS and an FkbH-like gene in S. griseus chromosome was disrupted by PCR-targeted gene disruption method (Gust et al. 2003). Firstly the disruption cassettes containing $5^{\prime}$ flanking upstream region and 3 '-flanking downstream region for gene disruption, two FRT sites, apramycin resistance gene for selection, and ori $\mathrm{T}$ for conjugation in the size of $1.4 \mathrm{~kb}$ were amplified using pIJ773 plasmid as a template (Additional file 1: Figure S3). Those amplified

(A)

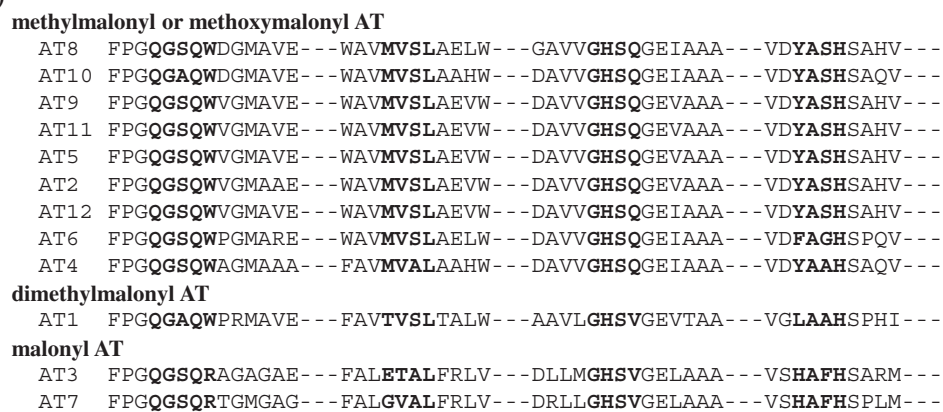

(B)

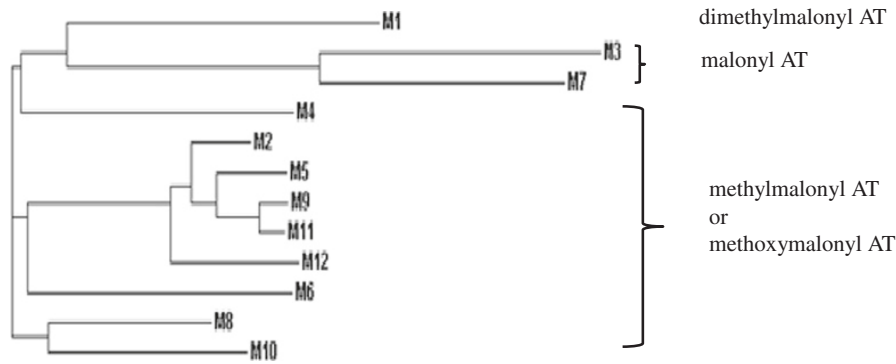

Figure 4 Comparison of nucleotide sequences at the active sites in each acyltransferase (AT) domain. (A) Sequence alignment of each domain by ClustalW. (B) Phylogenic dendrogram of each domain. The first AT domain in module 1 (AT1) was highly similar with AT domains specific for dimethylmalonyl-CoA to incorporate isobutyl group. Two AT domains of module 3 (AT3) and module 7 (AT7) was highly homologous with other AT domains specific for malonyl-CoA to incorporate acetyl group. The other nine AT domains exhibited the similar sequences with other AT domains specific for methylmalonyl-CoA to incorporate propionyl group. Contrastly, AT domains of module 6 (AT6) and module 12 (AT12) shows high similarity with AT domains specific for methylmalonyl-CoA, but they were assumed to be specific for methoxymalonyl-CoA to incorporate methoxyacetyl group, based on the chemical structure of bafilomycin. 
disruption cassettes were used for disruption of each gene in pSGB20 cosmid by homologous recombination in E. coli BW25113/pIJ790. The disrupted cosmid, pSGB20DH12-apr or pSGB20-FkbH-apr, was transformed again into E. coli ET12567 containing conjugal plasmid pUZ 8002. This E. coli strain was conjugated in the presence of $\mathrm{MgCl}_{2}$ with wild-type $S$. griseus spores. After 5 daycultivation, the positive conjugants, S. griseus DH12-apr or S. griseus $\mathrm{FkbH}$-apr, which was resistant to apramycin but sensitive to kanamycin were selected. The chromosomal DNAs of disrupted strains were confirmed by PCR analysis (Additional file 1: Figure S4).

After cultivation of wild strain and two disruptants, the antimicrobial activity on $R$. solani was examined. Even though the culture broth of wild strain showed the growth inhibitory activity, those of two deleted mutants, DH12apr and FkbH-apr did not (Figure 2A). The culture broths were extracted with ethyl acetate and the metabolites were purified sequentially through two different silica gel column chromatography. The HPLC analysis of extract from wild strain showed the typical peak for bafilomycin $B_{1}$ but no peak for bafilomycin $A_{1}$. However in the extracts of two disruptants, neither bafilomycin $A_{1}$ nor bafilomycin $\mathrm{B}_{1}$ was found in HPLC profiles (Figure $2 \mathrm{~B}$ ). The above results imply that the cloned gene cluster really commands the bafilomycin biosynthesis in S. griseus DSM 2608.

\section{Discussion}

Plecomacrolide antibiotic is an unusual macrolide antibiotic which has a structural element of 6-membered hemiacetal ring connected to the macrolactone ring. The unusual structure drew the scientist's interests about their biosynthetic pathway. Among plecomacrolide antibiotics, the concanamycin biosynthetic gene cluster was firstly reported from S. neyagawaensis (Haydock et al. 2005), and the biosynthetic pathway of plecomacrolide backbone was partly deduced.

We cloned an $87.4 \mathrm{~kb}$ chromosomal region of S. griseus DSM 2608, including presumable biosynthetic gene cluster of bafilomycin. This gene cluster was confirmed by metabolite analysis after gene disruption.

Among five type I PKS genes ranged in $58.5 \mathrm{~kb}$, the first type I PKS gene bafSI was comprised of 4 PKS modules, the second bafSII of 3 PKS modules, the third bafSIII of 2 PKS modules, the fourth bafSIV of 2 PKS modules, and the fifth bafSV of 1 PKS module having thioesterase domain (Figure 3). Thus one starter unit and eleven extender modules are involved in the biosynthesis of bafilomycin main backbone.

The substrate specificity of an acyltransferase (AT) domain of each module determines the incorporation of specific acyl-CoA precursors. In comparison of active sites of AT domains in this gene cluster showed that AT domain

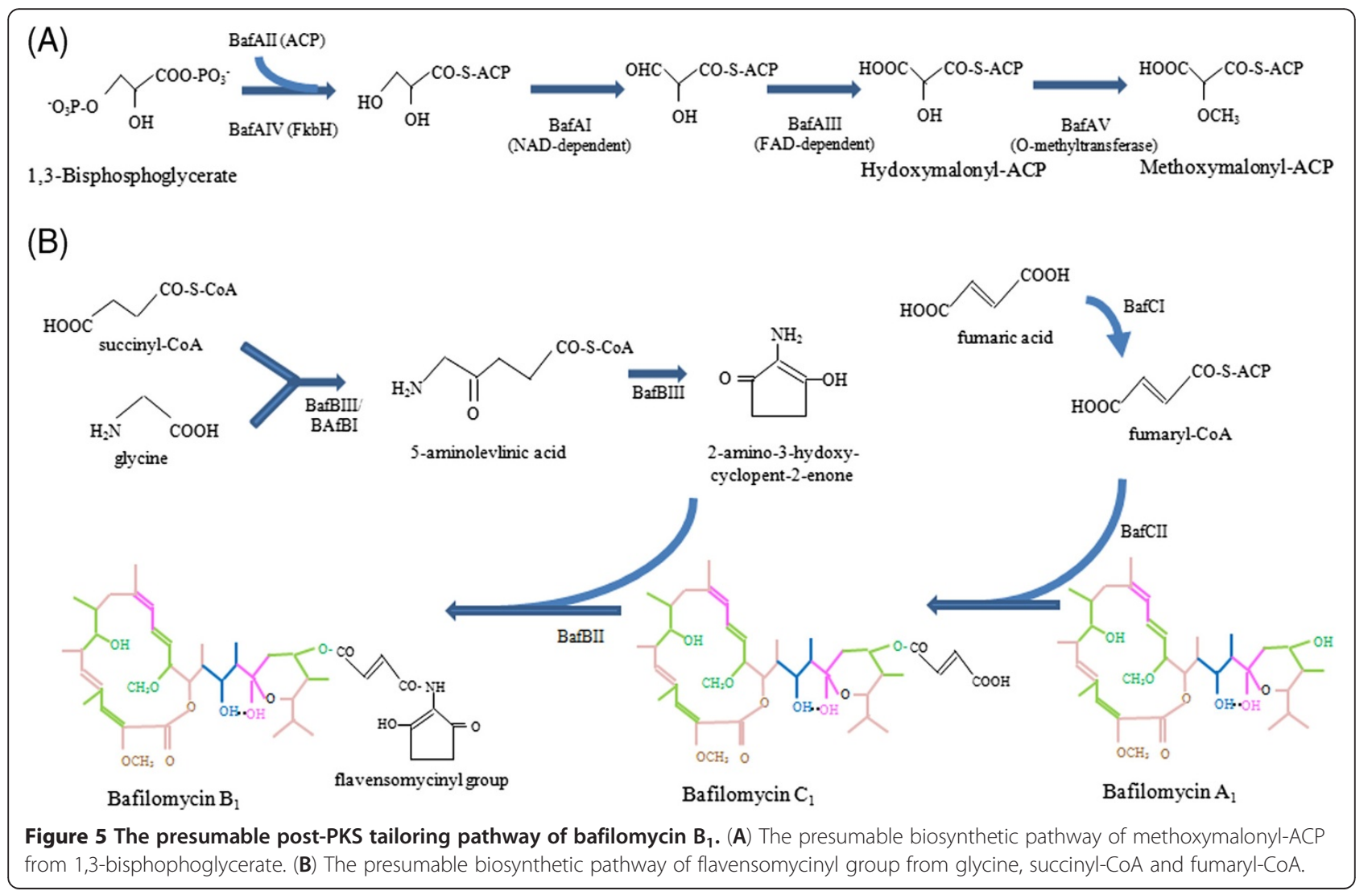


of the starter module 1 has the conserved sequences specific for dimethylmalonyl-CoA to incorporate isobutyl group (Figure 4). In contrast, AT domains of module 3 and module 7 has the high homology with other AT domains specific for malonyl-CoA to incorporate acetyl group. The other nine AT domains in the bafilomycin PKS genes exhibited the similar sequences with other AT domains specific for methylmalonyl-CoA to incorporate propionyl group. From chemical structure of bafilomycin, it can be deduced that AT domains of module 6 and module 12 might be specific for methoxymalonyl-CoA to incorporate methoxyacetyl group. However, any significant sequence differences between AT domains specific for methoxymalonyl-CoA and those for methylmalonyl-CoA was not found.

Thus the first PKS having 4 modules encoded from bafSI starts the polyketide synthesis from isobutyl group, and extend its length by incorporating 1 acetyl group and 2 propionyl groups, making 9 carbon backbone. Considering the chemical structure of bafilomycin, the hemiacetal ring can be formed at this step before further polyketide extension.

The polyketide biosynthesis is continuously mediated by following PKS proteins directed by BafSII, BafSIII, BafSIV, and the final BafSV. Those PKSs are comprised of 8 modules which can incorporate 1 acetyl group, 2-methoxyacetyl groups and 5 propionyl groups during polyketide extension. Since the incorporation of the methoxy groups at $\mathrm{C}-2$ and $\mathrm{C}-14$ position of bafilomycin macrolactone is originated from glycerol by feeding experiments of radiolabeled precursors (Schuhmann and Grond 2004; Schuhmann et al. 2007), it is imagined that module 6 and module 12 might transfer 2-methoxyacetyl group by methoxymalonyl-CoA originated from glycerol.

The generation of methoxymalonyl-CoA from 1,3bisphosphoglycerate was already proven in the biosynthesis of ansamitocin (Wenzel et al. 2006), zwittermicin (Chan and Thomas 2010) and tetronomycin (Sun et al. 2008). The fed glycerol is firstly converted into 1,3bisphosphoglycerate for primary metabolism, which can be acylated on acyl carrier protein (ACP) to produce glyceryl-ACP. This glyceryl-ACP is further oxidized to hydroxymalonyl-ACP by NAD-dependent acyl-CoA dehydrogenases and FAD-dependent acyl-CoA dehydrogenase. Finally methoxymalonyl-ACP is produced by methylation of hydroxymalonyl-ACP by O-methyltransferase (Figure 5A).

The compound made after macrolactone formation is bafilomycin $A_{1}$, which can be modified to bafilomycin $\mathrm{C}_{1}$ by esterification with fumaryl group at hydroxyl group in hemiacetal ring, and further to bafilomycin $B_{1}$ by linking 2-amino-3-hydroxy-cyclopenta-2-enone, finally building-up flavensomycinyl moiety (Figure 5B). In the bafilomycin biosynthetic gene cluster cloned here, the genes responsible for the biosynthesis of 2-amino-3hydroxy-cyclopenta-2-enone were also found. The 2-amino-3-hydroxy-cyclopenta-2-enone production through 2 steps of enzyme reaction was reported previously (Zhang et al. 2010). Firstly, 5-aminolevulinate synthase can catalyze the formation of 5-aminolevulinic acid from glycine and succinyl-CoA, which is then converted to 5aminolevulinyl-CoA by acyl-CoA ligase. Even though 5-aminolevulinyl-CoA is spontaneous converted to 2,5piperidinedione due to its instability, it is more easily cyclized to 2-amino-3-hydroxy-cyclopenta-2-enone in the presence of 5-aminolevulinate synthase. Finally this compound can be connected with fumaryl group by amide synthetase.

For the incorporation of fumaryl group into flavensomycinyl moiety, it was assumed that fumaric acid is firstly converted to fumaryl-CoA by BafCI (acyl CoA ligase), and then connected to bafilomycin polyketde backbone by BafCII (malonyl transferase). However, the catalytic properties of those enzymes should be further characterized.

\section{Additional file}

\begin{abstract}
Additional file 1: Figure S1. The amplified products of KS, ALA, and FkbH probes. Lane M, 1 kb DNA ladder; lane 1, the amplified KS probe (590 bp); lane 2, the amplified FkbH probe (600 bp); lane 3, the amplified ALS probe (520 bp). Figure S2. Screening of bafilomycin biosynthetic gene cluster. pSGB1 cosmid clone was selected based on the multiple bands binding with KS probe. pSGB20 cosmid clone was isolated by using KS probe and FkbH probe, and pSGB23 cosmid clone was screened by using KS probe and ALS probe. (A) agarose gel electrophoresis pattern; (B) Southern blotting result. Left lane, 1 kb DNA ladder; right lane, each cosmid DNA digested with Ncol. Figure S3. The amplified disruption cassettes for gene inactivation for $\mathrm{DH}$ domain in PKS module 12 and FkbH gene. Lane M, 1 kb DNA ladder; lane 1, the amplified disruption cassette for DH domain of PKS module 12 (1.3 kb); lane 2, the amplified disruption cassette for FkbH gene (1.3 kb). Figure S4.

Confirmation of disrupted mutants by gene amplification from chromosomal DNA. (A) Confirmation of DH domain of PKS module 12. Lane M, 100 bp DNA ladder; lane 1, the amplified gene from plJ773 plasmid (template for disruption cassette); lane 2, the amplified DNA from the wild strain chromosome (1661 bp); lane 3, the amplified DNA from the disrupted mutant chromosome (1652 bp). (B) Confirmation of FkbH gene. Lane M, 100 bp DNA ladder; lane 1, the amplified DNA from plJ773 plasmid (template for disruption casstte); lane 2, the amplified DNA from the wild strain chromosome (1280 bp); lane 3, the amplified DNA from the disrupted mutant chromosome (1554 bp).
\end{abstract}

\section{Competing interests}

The authors declare that they have no competing interests.

\section{Acknowledgement}

This work was supported by Korea Research Fund (grant number NRF-2011-0006078).

Received: 24 April 2013 Accepted: 2 May 2013

Published: 10 May 2013

\section{References}

Bierman M, Logan R, O'Brien K, Seno ET, Rao RN, Schoner BE (1992) Plasmid cloning vectors for the conjugal transfer of DNA from Escherichia coli to Streptomyces spp. Gene 116:43-49 
Bowman EJ, Sibers A, Aldendorf K (1988) Bafilomycins: A class of inhibitors of membrane ATPases from microorganisms, animal cells and plant cells. Proc Natl Acad Sci USA 85:7972-7976

Chan YA, Thomas MG (2010) Recognition of (2S)-aminomalonyl-acyl carrier protein (ACP) and (2R)-hydroxymalonyl-ACP by acyltransferases in zwittermicin A biosynthesis. Biochemistry 49:3667-3677

Datsenko KA, Wanner BL (2000) One-step inactivation of chromosomal genes in Escherichia coli K-12 using PCR products. Proc Natl Acad Sci USA 97:6640-6645

Dröse S, Altendorf K (1997) Bafilomycins and concanamycins as inhibitors of V-ATPases and P-ATPases. J Exp Biol 200:1-8. 1997

Farina C, Gagliardi S (2002) Selective inhibition of osteoclast vacuolar H (+)-ATPase. Curr Pharm Design 8:2033-2048

Gust B, Challis GL, Fowler K, Kieser T, Chater KF (2003) PCR-targeted streptomyces gene replacement identifies a protein domain needed for biosynthesis of the sesquiterpene soil odor geosmin. PNAS 100:1541-1546

Hagenmaier H-P, Werner G, Drautz H, Holst H, Zähner H, Brandes W, Reinecke P, Zoebelein G, Stendel W, Andrews P, Schaller K (1985) Bafilomycin pesticide. US Patent. 4,558,139

Haydock SF, Appleyard AN, Mironenko T, Lester J, Scott N, Leadlay PF (2005) Organization of the biosynthetic gene cluster for the macrolide concanamycin A in Streptomyces neyagawaensis ATCC 27449. Microbiology 151:3161-3169

Ichikawa N, Oguchi A, Ikeda H, Ishikawa J, Kitani S, Watanabe Y, Nakamura S, Katano Y, Kishi E, Sasagawa M, Ankai A, Fukui S, Hashimoto Y, Kamata S, Otoguro M, Tanikawa S, Nihira T, Horinouchi S, Ohnishi Y, Hayakawa M, Kuzuyama T, Arisawa A, Nomoto F, Miura H, Takahashi Y, Fujita N (2010) Genome sequence of Kitasatospora setae NBRC 14216: An evolutionary snapshot of the family Streptomycetaceae. DNA Res 17:393-406

Ishikawa J, Hotta K (1999) Frame plot: a new implementation of the frame analysis for predicting protein-coding regions in bacterial DNA with high $\mathrm{G}$ $+C$ content. FEMS Microbiol Lett 174:251-253

Kieser T, Bibb MJ, Buttner MJ, Chater KF, Hopwood DA (2000) Practical Streptomyces Genetics. The John Innes Foundation, Norwich, UK

Kinashi H, Someno K, Sakaguchi K (1984) Isolation and characterization of concanamycin A, B and C. J Antibiot 37:2509-2511

Moon S-S, Hwang W-H, Chung YR (2003) New cytotoxic bafilomycin $C_{1}$-amide produced by Kitasatospora cheerisanensis. J Antibiot 56:856-861

Ohta E, Kubota NK, Ohta S, Suzuki M, Ogawa T, Yamasaki A, Ikegami S (2001) Micromonospolides A-C, new macrolides from Micromonospora sp. Tetrahedron 57:8463-8467

O'Shea MG, Rickards RW, Rothschild JM, Lacey E (1997) Absolute configurations of macrolide antibiotics of the bafilomycin and leucanicidin groups. $J$ Antibiot 50:1073-1077

Otoguro K, Nakagawa A, Omura S (1988) Setamycin, a 16-membered macrolide antibiotic. Identification and nematocidal activity. J Antibiot 41:250-252

Sambrook J, Fritsch EF, Maniatis T (1989) Molecular Cloning: A Laboratory Manual. CSH Press, NY, USA

Seto H, Tajima I, Akao H, Furihata K, Otake N (1984) The isolation and structures of hygrolidin amide and defumarylhygrolidin. J Antibiot 37:610-613

Shacka JJ, Klocke BJ, Roth KA (2006) Autophagy, bafilomycin and cell death. The "A-B-Cs" of plecomacrolide-induced neuroprotection. Autophagy 2:228-230

Schuhmann T, Grond S (2004) Biosynthetic investigation of the V-type ATPase inhibitors bafilomycin $A_{1}, B_{1}$ and concanamycin A. J Antibiot 57:655-661

Schuhmann T, Vollmar D, Grond S (2007) Biosynthetic origin of the methoxy extender unit in bafilomycin and concanamycin using stereospecifically labeled precursors. J Antibiot 60:53-60

Sun Y, Hong H, Gillies F, Spencer JB, Leadlay PF (2008) Glyceryl-S-acyl carrier protein as an intermediate in the biosynthesis of tetronate antibiotics. ChemBioChem 9:150-156

Wenzel SC, Williamson RM, Grünanger C, Xu J, Gerth K, Martinez RA, Moss SJ, Carroll BJ, Grond S, Unkefer CJ, Müller R, Floss HG (2006) On the biosynthetic origin of methoxymalonyl-acyl carrier protein, the substrate for incorporation of "glycolate" units into ansamitocin and soraphen A.J Am Chem Soc 128:14325-14336

Werner G, Hagenmaier H, Drautz H, Baumgartner A, Zähner H (1984) Metabolic products of microorganisms. 224. Bafilomycins, a new group of macrolide antibiotics. Production, isolation, chemical structure and biological activity. J Antibiot 37:110-117
Xu J, Cheng T, Feng HT, Pavlos NJ, Zheng MH (2007) Structure and function of V-ATPases in osteoclasts: potential therapeutic targets for the treatment of osteolysis. Histol Histopathol 22:443-454

Zhang W, Bolla ML, Kahne D, Walsh CT (2010) A three enzyme pathway for 2-amino-3-hydroxycyclopent-2-enone formation and incorporation in natural product biosynthesis. J Am Chem Soc 132:6402-6411

doi:10.1186/2191-0855-3-24

Cite this article as: Hwang et al:: Organization and characterization of a biosynthetic gene cluster for bafilomycin from Streptomyces griseus DSM 2608. AMB Express 2013 3:24

\section{Submit your manuscript to a SpringerOpen ${ }^{\odot}$ journal and benefit from:}

- Convenient online submission

- Rigorous peer review

- Immediate publication on acceptance

- Open access: articles freely available online

- High visibility within the field

- Retaining the copyright to your article

Submit your next manuscript at $>$ springeropen.com 